\title{
Quantification of myocardial blood flow with dynamic SPECT acquisitions: ready for prime time?
}

\author{
Fabien Hyafil ${ }^{1}$. François Rouzet ${ }^{1}$. Dominique Le Guludec ${ }^{1}$
}

Published online: 25 August 2018

(C) Springer-Verlag GmbH Germany, part of Springer Nature 2018

\section{Quantification of MBF with cardiac-centred gamma cameras}

Thanks to PET studies, the measurement of global and regional stress and rest blood flow and flow reserve has proven to be of diagnostic and prognostic value in various clinical settings. A growing number of nuclear medicine sites in Europe are now using cardiac-centred gamma cameras for myocardial perfusion imaging (MPI). In so-called "CZT cameras" a cadmium-zinctelluride (CZT) crystal is used in place of the conventional sodium/ iodine $(\mathrm{Na} / \mathrm{I})$ crystal for the detection of gamma rays. Manufacturers have taken advantage of these much thinner and more flexible CZT detectors to design gamma cameras dedicated to cardiac imaging that offer a larger surface for signal detection and focused on the heart region [1].

The tomographic sensitivity of a gamma camera system for myocardial counts is a critical parameter that influences the minimal duration of SPECT acquisitions for reconstruction of 3D images of the heart. The tomographic sensitivity of conventional gamma cameras with $\mathrm{Na} / \mathrm{I}$ detectors is relatively low with only a few parts-per-million of the injected activity being detected within the myocardial area, but the sensitivity of CZT gamma cameras is four to seven times higher $[2,3]$. The higher sensitivity of the CZT camera allows the reconstruction of 3D cardiac SPECT acquisitions with relatively short time frames and reasonable spatial resolution, and has renewed interest in dynamic cardiac imaging with SPECT. The aim of dynamic cardiac acquisitions is to detect the first-pass blood perfusion of tracer and its extraction

This Editorial Commentary refers to the article https://oi.org/10.1007/ s00259-018-4057-5.

Dominique Le Guludec

dominique.leguludec@aphp.fr

1 Department of Nuclear Medicine, Centre Hospitalier Universitaire Bichat, Assistance Publique - Hôpitaux de Paris, Département Hospitalo-Universitaire FIRE, Inserm 1148, Université Paris Diderot, 46 rue Henri Huchard, 75018 Paris, France into the myocardium to allow quantification of myocardial blood flow (MBF) using dedicated compartment models.

Recently, the ability of protocols with ${ }^{99 \mathrm{~m}} \mathrm{Tc}$-labelled perfusion tracers and CZT SPECT to quantify stress and rest MBF has been demonstrated [4], showing relatively good correlations between myocardial flow reserve (MFR) values, stress to rest MBF ratios estimated using SPECT imaging and those measured using PET imaging with ${ }^{15} \mathrm{O}$-water [5] or ${ }^{13} \mathrm{~N}$-ammonia [6] as perfusion tracers. In this issue of EJNMMI, Han et al. [7] describe the feasibility of a dual isotope $\left({ }^{201} \mathrm{Tl} /{ }^{99 \mathrm{~m}} \mathrm{Tc}\right)$ dynamic imaging protocol for the quantification of MBF using CZT SPECT.

\section{Interest in SPECT-based quantification of MBF}

PET procedures for the quantification of MBF have been well validated and provide accurate measurements of stress and rest MBF and MFR. However, PET MPI represents only a small proportion of MPI scans performed worldwide because of the limited access to PET perfusion tracers that require either an onsite cyclotron or an expensive ${ }^{82} \mathrm{Rb}$ generator, and the limited time available for dedicated cardiovascular imaging on most clinical PET scanners. In contrast, dynamic SPECT acquisitions benefit from the low cost and wide availability of SPECT perfusion tracers and the shorter acquisition times for conventional MPI using the CZT camera. The rationale for the dual isotope approach chosen by Han et al. [7] is that Tl has a higher firstpass myocardial extraction rate and less roll-off at high flow than Tc-labelled perfusion tracers and therefore allows more accurate evaluation of MBF during stress.

For rest dynamic acquisitions, the choice of Tc-labelled perfusion tracers is relevant because rest images can be acquired without any background signal in the myocardium thanks to the different energy emission ranges of $\mathrm{Tl}$ and $\mathrm{Tc}$, and under conditions of low flow for which the myocardial extraction of Tc-labelled perfusion tracer remains linear. Using this dual isotope dynamic SPECT imaging protocol in 34 patients, Han et al. [7] demonstrated the feasibility of measuring global and regional MBF and 
found good correlations between MFR values and the anatomical and functional severity of coronary stenosis evaluated invasively. Although the results of this proof-of-concept study are promising, the road towards the integration of SPECT-based MBF quantification in the clinical interpretation of MPI is still long. In this Editorial, we discuss the technical challenges and potential clinical applications of SPECT-based quantification of MBF.

\section{Technical challenges of SPECT-based quantification of MBF}

PET-based myocardial flow quantification has demonstrated good test-retest repeatability for stress MBF and MFR measurements with a day-to-day coefficient of variation of less than $20 \%$ and reproducibility among laboratories $(<10 \%$ variation when using similar methods) [8-10]. There are, however, a number of technological issues that might prevent SPECTbased myocardial flow quantification reaching such a high but desired level of reproducibility for MBF measurements.

First, the prerequisite for accurate quantitative estimation of myocardial flow using compartment modelling is to obtain a reliable activity curve for the blood first-pass with good separation between the blood and myocardial activity over time. The spatial resolution of SPECT images is lower than that of PET images, and that of dynamic 3D SPECT images with short acquisition times is even lower. The robustness of SPECTbased MBF measurements might be affected by worse separation between activities originating from the left ventricle (LV) cavity and the myocardium. Second, the relatively low-energy of X-ray emissions of $\mathrm{Tl}(70-80 \mathrm{keV})$ results in a strong Compton scatter signal that degrades the contrast and quality of reconstructed images. Third, CT-based correction of tissue attenuation with SPECT is intrinsically less accurate than with PET. In addition, the use of two isotopes with different photon energies and tissue attenuation properties might induce heterogeneities in the estimated values for regional MBF extracted from dynamic SPECT acquisitions.

Additional clinical studies are required to evaluate the robustness, reproducibility, homogeneity and accuracy of regional and global MBF measurements obtained from dynamic SPECT imaging protocol. This information is critical to the more precise understanding of the potential role of SPECTbased MBF measurements in the clinical interpretation of MPI.

\section{Clinical value of adding global MBF quantification to SPECT MPI?}

Dynamic SPECT acquisition protocols require longer times under the camera and are more complex than conventional MPI protocols. Consequently, before implementation of these protocols can be justified, improvements in the diagnostic and prognostic performance of SPECT MPI are needed. Based on the large clinical experience acquired with cardiac PET imaging, it can be assumed that SPECT-based quantification of global MBF could provide added value for the interpretation of MPI in several clinical situations. First, an important limitation of SPECT MPI is that regional myocardial uptake of perfusion tracers is evaluated only relative to the highest signal detected in the myocardium and may fall short in identifying balanced myocardial ischaemia in patients with triple-vessel CAD. Measurements of low global stress MBF or MFR on dynamic cardiac SPECT could help identify patients with triple-vessel $\mathrm{CAD}$, in particular in the presence of high-risk features of extensive myocardial ischaemia on conventional SPECT MPI such as stress LV dysfunction, transient ischaemic dilatation and increased right ventricular uptake, or alternatively exclude triple-vessel CAD if global MFR is preserved.

Second, an important concern when using a pharmacological stress test is the risk of hidden ingestion or persistent circulation of products, in particular caffeine, that antagonize the effects of adenosine on its receptors resulting in the absence or reduced stimulation of myocardial hyperaemia. In this situation, the haemodynamic impact of coronary stenosis is underestimated and MPI might be falsely interpreted as normal. MBF quantification could help identify nonresponders to pharmacological stress on SPECT MPI who will not show any change in global MBF values during stress. Third, the addition of the global MFR value measured with $\mathrm{Rb}$ PET MPI has been found to have an incremental value over the extent of myocardial ischaemia for predicting the risk of cardiovascular events during follow-up [11, 12]. In a similar way to what has been described with Rb PET, it can be hypothesized that SPECT-based MFR values could help improve the stratification of the cardiovascular risk of patients.

\section{Clinical value of adding regional MBF quantification to SPECT MPI?}

The effort to move from relative to absolute regional MBF quantification is motivated by the assumption that flow quantification could provide a more precise and reliable assessment of the haemodynamic impact of coronary stenosis in an approach similar to the measurement of fractional flow reserve (FFR) during invasive coronary angiography. The implementation of absolute stress MBF or MFR for the evaluation of coronary stenosis, however, might face some significant hurdles. First, SPECTbased regional MBF values need to be homogeneous and comparable within the LV to define consistent threshold values for myocardial ischaemia that can apply to all myocardial territories. The technological limitations of SPECT-based quantification of MBF described above might make it difficult to achieve sufficient signal homogeneity for this analysis. Second, several factors other than flow-limiting stenosis of the epicardial coronary 
arteries can result in a decrease in stress MBF and MFR, including diffuse atherosclerotic disease, cardiac microvascular dysfunction, capillary rarefaction and endothelial dysfunction. Han. et al. [7] found only a moderate correlation between regional MFR quantified noninvasively with dynamic SPECT and FFR values measured invasively. This finding is, however, consistent with previous results obtained using Rb PET [13].

The interpretation of absolute stress MBF is complex. The respective roles of coronary stenosis and microvascular diseases in the reduction of myocardial flow can only be evaluated by analysis of stress MBF values in combination with the results of invasive coronary angiography and FFR measurements [14]. For simplification, it can be assumed that cardiac microvascular diseases predominantly affect global MFR, whereas coronary stenosis mostly causes a regional reduction in MFR. An easy way to correct for the effects of microvascular disease is thus to adjust the regional flow values to the maximal value of stress MBF measured within the LV. This is exactly what is done for semiquantitative interpretation of MPI, but at the cost of losing information on the effects of diffuse CAD and microvascular disease on stress MBF. Han et al. [7] found strong correlations between summed stress scores based on the semiquantitative analysis of MPI and the regional MFR values obtained from dynamic cardiac acquisitions. These results clearly raise the question as to the additional clinical value of regional MFR quantification for the clinical interpretation of MPI.

\section{Conclusion}

The study by Han et al. [7] confirms the feasibility of SPECTbased quantification of MBF using dynamic perfusion imaging. The robustness and reproducibility of these measurements need to be confirmed in multicentre studies with larger numbers of patients. MBF measurements with dynamic SPECT protocols might not achieve the accuracy and robustness of PET imaging, but could offer access to estimates of MFR in a larger number of sites and at lower cost. The analysis of global MFR values seems easier to integrate into the clinical interpretation of MPI than regional MFR because of the lower signal homogeneity of SPECT in comparison to PET perfusion images. This additional information, though not perfectly accurate, might help overcome some of the current limitations of SPECT MPI related to the semiquantitative analysis of radiotracer uptake in the myocardium.

\section{References}

1. Agostini D, Marie PY, Ben-Haim S, Rouzet F, Songy B, Giordano A, et al. Performance of cardiac cadmium-zinc-telluride gamma camera imaging in coronary artery disease: a review from the cardiovascular committee of the European Association of Nuclear Medicine (EANM). Eur J Nucl Med Mol Imaging. 2016;43:2423-32.

2. Imbert L, Poussier S, Franken PR, Songy B, Verger A, Morel O, et al. Compared performance of high-sensitivity cameras dedicated to myocardial perfusion SPECT: a comprehensive analysis of phantom and human images. J Nucl Med. 2012;53:1897-903.

3. Verger A, Imbert L, Yagdigul Y, Fay R, Djaballah W, Rouzet F, et al. Factors affecting the myocardial activity acquired during exercise SPECT with a high-sensitivity cardiac CZT camera as compared with conventional Anger camera. Eur J Nucl Med Mol Imaging. 2014;41:522-8.

4. Ben-Haim S, Murthy VL, Breault C, Allie R, Sitek A, Roth N, et al. Quantification of myocardial perfusion reserve using dynamic SPECT imaging in humans: a feasibility study. J Nucl Med. 2013;54:873-9.

5. Agostini D, Roule V, Nganoa C, Roth N, Baavour R, Parienti JJ, et al. First validation of myocardial flow reserve assessed by dynamic (99m)Tc-sestamibi CZT-SPECT camera: head to head comparison with (15)O-water PET and fractional flow reserve in patients with suspected coronary artery disease. The WATERDAY study. Eur J Nucl Med Mol Imaging. 2018;45:1079-90.

6. Nkoulou R, Fuchs TA, Pazhenkottil AP, Kuest SM, Ghadri JR, Stehli J, et al. Absolute myocardial blood flow and flow reserve assessed by gated SPECT with cadmium-zinc-telluride detectors using 99mTc-tetrofosmin: head-to-head comparison with $13 \mathrm{~N}$ ammonia PET. J Nucl Med. 2016;57:1887-92.

7. Han S, Kim YH, Ahn JM, Kang SJ, Oh JS, Shin E, et al. Feasibility of dynamic stress 201Tl/rest 99mTc-tetrofosmin single photon emission computed tomography for quantification of myocardial perfusion reserve in patients with stable coronary artery disease. Eur J Nucl Med Mol Imaging. 2018. https://doi.org/10.1007/ s00259-018-4057-5.

8. Klein R, Renaud JM, Ziadi MC, Thorn SL, Adler A, Beanlands RS, et al. Intra- and inter-operator repeatability of myocardial blood flow and myocardial flow reserve measurements using rubidium82 PET and a highly automated analysis program. J Nucl Cardiol. 2010;17:600-16.

9. El Fakhri G, Kardan A, Sitek A, Dorbala S, Abi-Hatem N, Lahoud $\mathrm{Y}$, et al. Reproducibility and accuracy of quantitative myocardial blood flow assessment with (82)Rb PET: comparison with (13)Nammonia PET. J Nucl Med. 2009;50:1062-71.

10. Kitkungvan D, Johnson NP, Roby AE, Patel MB, Kirkeeide R, Gould KL. Routine clinical quantitative rest stress myocardial perfusion for managing coronary artery disease: clinical relevance of test-retest variability. JACC Cardiovasc Imaging. 2017;10:565-77.

11. Farhad H, Dunet V, Bachelard K, Allenbach G, Kaufmann PA, Prior JO. Added prognostic value of myocardial blood flow quantitation in rubidium- 82 positron emission tomography imaging. Eur Heart J Cardiovasc Imaging. 2013;14:1203-10.

12. Ziadi MC, Dekemp RA, Williams KA, Guo A, Chow BJ, Renaud $\mathrm{JM}$, et al. Impaired myocardial flow reserve on rubidium- 82 positron emission tomography imaging predicts adverse outcomes in patients assessed for myocardial ischemia. J Am Coll Cardiol. 2011;58:740-8.

13. Juneau D, deKemp RA, Beanlands RSB. Reporting myocardial flow reserve with PET. Ready or not, here it is! But walk before you fly! J Nucl Cardiol. 2018;25:164-8.

14. Gould KL, Johnson NP, Bateman TM, Beanlands RS, Bengel FM, Bober R, et al. Anatomic versus physiologic assessment of coronary artery disease. Role of coronary flow reserve, fractional flow reserve, and positron emission tomography imaging in revascularization decision-making. J Am Coll Cardiol. 2013;62:1639-53. 